\title{
Qualitative Land Suitability Evaluation for the Growth of Rice and Off-seasons Crops as Rice Based Cropping System on Paddy Fields of Central Guilan, Iran
}

\author{
Shahram Mahmoud Soltani ${ }^{1^{*}}$, M. M. Hanafi, ${ }^{2,3}$ M. T. Karbalaei ${ }^{3}$ and B. Khayambashi ${ }^{3}$ \\ 'Laboratory of Food Crops, Institute of Tropical Agriculture, Universiti Putra Malaysia, 43400, \\ Serdang, Selangor, Malaysia; shmsoltani@gmail.com \\ ²aboratory of Plantation Crops, Institute of Tropical Agriculture, Universiti Putra Malaysia, 43400, \\ Serdang, Selangor, Malaysia; mmhanafi@agri.upm.edu.my \\ ${ }^{3}$ Department of Land Management, Faculty of Agriculture, Universiti Putra Malaysia, 43400, \\ Serdang, Selangor, Malaysia; mtkarbalai2@yahoo.com, bkhayam@yahoo.com
}

\begin{abstract}
Land suitability evaluation analysis is a necessecary to achieve optimum management and utilization of the available land resources for sustainable agricultural crops production. One of the most serious and urgent constraint in lowland paddy fields of Iran is to improve agricultural land management and cropping patterns either to increase the agricultural production with proper land use resources or to get more income from out of season plant as a second crops.Therefore, the objective of this study was to find the most suitable off-season crops after rice. Qualitative Land Suitability (QLS) classification of fifteen first and second agricultural crops in the paddy fields of the Guilan plain in Iranunder RBCS that covers an area of 40567 ha was carried out. By using the semi-detailed method of soil classification, 24 soil unites at 11 soil series in five different physiographic units were indentified (river alluvial plains ( 5 series), low lands (2 series), river banks (2 series) and coastal plains and sand dunes (2 series)). Land and climate characteristics of each crop with its requirements were also determined and rated based upon Parametric and simple limitation method proposed by Sys et al. [1]. The obtained indexes indicated that land suitability classes varied from $\mathrm{S}_{2}$ (moderately suitable) to $\mathrm{N}$ (Not suitable). The highest land suitability class for crops are: $S_{2}$ for Rice, Spinach, Beans, Radish, Garlic, Shall cress, Clover, Lettuce, Rape seed, Bean (vulgaris and faba cultivars) and $\mathrm{S}_{3}$ for Wheat, Carrot, Barley, Triticale, and Rice (Ratoon). The most important limiting factors are: climate (low temperature and high rainfall at vegetative and reproductive growth stages), drainage (shallow water table depth), soil (texture) and fertility (low organic carbon content, $\mathrm{pH}$ ) (alone or in combination) respectively, those influence adversely on the land suitability classes, especially climatic and drainage limitation, because of growing season in autumn and winter Verification of the factors and their relative significance is very important for decision on land suitability.
\end{abstract}

Keywords: Land Suitability, Paddy Fields, Rice, Second Crops.

\section{Introduction}

Agricultural resources are considered to be one of the most important renewable and dynamic natural resources. Land and soil as basic portions of agricultural resources and economy are the ultimate sources of wealth andthe foundation on which civilization is constructed. A major problem of these agriculture resources

*Corresponding author:

Sh. Mahmoud Soltani (shmsoltani@gmail.com) 
is inappropriate land use which leads to inefficient exploitation of natural resources, destruction of land resources, poverty and other social problems and finally, destruction of civilization [2]. Consequently, the productivity of the land is declined and the country could not produce as much food as needed for the increasing population, especially for economic life of farmers. In particular, the rice production section as a strategic agricultural crops in Iran does not meet the food demands of rapidly growing country population and the farmers economic life. Similar to many developing countries, Iran is facingunsustainable and uneconomic land use. Therefore, there is an urgent need to use land in the most scientific and sustainable ways. A part of the problem can be solved by land suitability evaluation leading to rational land use planning [3], and appropriate and sustainable use of natural and human resources and also optimizing the use of a land piece for a specified use [4]. Land suitability is defined as the suitability of a given type of land to support a defined land use, either in its current state or after improvements. Land suitability evaluation described the process of appraisal and grouping of specific areas of land in terms of their suitability for defined uses [21]. Special land productivity strongly depends on climate, soil, topography and water availability, which are the most important categories of environmental information required for judging land suitability and microorganisms activity $[19,22]$. In fact, land suitability evaluation is an examination process of the degree of land suitability for a specific utilization type [23] and/or description method or estimation of potential land productivity [5]. In this manner, all requirements can be provided at the present time and also a suggestion can be made to meet the future population needs can be made, which is one ofthe basic principles of precision agriculture [6]. Rice as a strategic crop is important and commercially produced in the majority of coastal plains of Guilan province but it is a single crop and economically, the farmers need some other second cropsin Rice Based Cropping System (RBCS) for more income. Land suitability evaluation for some of these crops in the north of Iran coastal plains has been done by Mahmoud Soltani [7], Torabi [8], Khaksarian [9] and Akef [10] and in other parts of the world by Kuria et al. [11] and Ismail [12].

Therefore, the objectives of this Study not only confirms some obtained results about the previous researches findings of RBCS in paddy fields, but also presents a land suitability based upon simple limitation and parametric (root square) method to evaluate the capabilities of the study area for rice and the below mentioned second crops in RBCS in paddy fields. Hence, the methodology adopted in this study combines most aspects of climate, land and soil characteristics that influence crop suitability of the study area.

\section{Material and Methods}

\subsection{Study Area}

The present study was conducted in an extent of 40567 ha in central Guilan-North of Iran-for the period of 2009-2010.The area is located at $30 \mathrm{~km}$ northeast of Rasht (Capital of Guilan province), between $37^{\circ} 7^{\prime} 30^{\prime \prime}$ to $37^{\circ} 27^{\prime} 8^{\prime \prime}$ North longitudes and $49^{\circ} 31^{\prime} 00^{\prime \prime}$ to $49^{\circ} 45^{\prime} 00^{\prime \prime}$ East latitudes. The altitude of the region is $-28 \mathrm{~m}$ a.s.l. It is bordered to the north by Caspian Sea, to the south by water channel of Rasht-Sangar, to the east by Gayshe-damerde River, and to the west by Siahrood River. The entire study area belongs to Sepidrood delta where its topography is almost flat with less than $1 \%$ slope. Most of the study area are planted to irrigated rice in spring and summer, and all aforementioned second crops are planted, based on farmer decision, after rice has been harvested .

\subsection{Climate of the Study Area}

The most important climate characteristics (Table 2) necessary for suitability determination (temperature, rainfall, relative humidity, etc.) were collected from Rasht synoptic metrological station. The study area has a mean annual rainfall of $1294 \mathrm{~mm}$ that is concentrated in autumn and winter seasons, with minimum and maximum relative humidity of $76.4 \%$ and $86.4 \%$, respectively and a mean annual temperature of $15.8^{\circ} \mathrm{C}$. The warmest month of the year is Tir (11 June- 11 July) with a mean of maximum temperature of $28.81^{\circ} \mathrm{C}$ and the coldest month of year is Day (from October - to early January) when the minimum temperature is as low as $4.24^{\circ} \mathrm{C}$. The annual potential evapo-transpiration reaches value of $720 \mathrm{~mm} \mathrm{[10].} \mathrm{According} \mathrm{to} \mathrm{the}$ climatic data and umberothermic curve, soil moisture regimes, soil temperature regime and climate are Udic and Aquic, thermic and very humid, respectively (based on Amberje climate classification). 


\subsection{Soil Classification}

In order to have more authentic soil data, particularly to control the soil boundaries and to up-date the soil characterics, the 24 soil unites at 11 soil series in 5 physiographic units (river alluvial plains (5 series), low lands (2 series),

Table 1. Land Suitability Index (LSI) and suitability class for a Land Use Type (LUT)

\begin{tabular}{rcl}
\hline \multicolumn{1}{c}{ LSI } & $\begin{array}{l}\text { Symbol } \\
\text { Suitability }\end{array}$ & Class \\
\hline $85-100$ & $\mathrm{~S}_{1}$ & Highly suitable \\
$60-85$ & $\mathrm{~S}_{2}$ & Moderately suitable \\
$40-60$ & $\mathrm{~S}_{3}$ & Marginally suitable \\
$25-40$ & $\mathrm{~N}_{1}$ & Currently not suitable \\
$0-25$ & $\mathrm{~N}_{2}$ & Permanently not suitable \\
\hline
\end{tabular}

Source: Sys C et al. [1]. river banks ( 2 series) and coastal plainsand dunes ( 2 series) in detailed soil survey) were selected. The profile description of pedons was made using standard terminology [13] after preparing and analyzing the samples [14] (Table 1). According to the USDA soil taxonomy system [15], the soils were classified as Entisols, Inceptisols and Alfisols orders (Table 3 ).

\subsection{Land Suitability Evaluation}

Land evaluation system developed by Sys et al. (1991) was used in the quantitative assessment of the agro-ecological evaluation in the study area for the land use types ( LUTs) of field crop. A digital soil database was prepared by entering the diagnostic characteristics (texture, slope, erosion, stoniness, depth, calcium carbonate, organic matter, $\mathrm{pH}$,

Table 2. Climatic characteristic of the synoptic meteorological station of Rasht (50years)

\begin{tabular}{lccccccc}
\hline Parameter & $\begin{array}{c}\text { Sunshine } \\
(\text { hours })\end{array}$ & $\begin{array}{c}\text { Mean max T } \\
\left({ }^{\circ} \mathrm{C}\right)\end{array}$ & $\begin{array}{c}\text { Mean min t } \\
\left({ }^{\circ} \mathrm{C}\right)\end{array}$ & $\begin{array}{c}\text { Mean month } \\
\mathrm{T}\left({ }^{\circ} \mathrm{C}\right)\end{array}$ & $\begin{array}{c}\text { Relative } \\
\text { humidity }(\%)\end{array}$ & $\begin{array}{c}\text { Rainfall } \\
(\mathrm{mm})\end{array}$ & $\begin{array}{c}\text { Calculated ET }_{\mathrm{p}} \\
(\mathrm{mm})\end{array}$ \\
\hline Jan & 2.69 & 9.55 & 4.52 & 7.0 & 85.4 & 134.9 & - \\
Feb & 2.84 & 9.29 & 4.24 & 6.8 & 85.7 & 118.7 & - \\
Mar & 2.77 & 11.08 & 6.58 & 8.9 & 85.1 & 109.4 & - \\
Apr & 4.32 & 15.93 & 10.74 & 13.45 & 81.0 & 61.3 & 60.4 \\
May & 5.7 & 21.28 & 16.06 & 18.7 & 78.2 & 53.7 & 87.9 \\
Jun & 7.11 & 26.03 & 19.98 & 23.0 & 76.5 & 44.9 & 119.5 \\
Jul & 7.29 & 28.81 & 22.60 & 25.7 & 76.4 & 41.5 & 132.7 \\
Aug & 5.63 & 28.34 & 22.38 & 25.4 & 79.0 & 72.7 & 106.9 \\
Sep & 4.73 & 25.7 & 19.61 & 22.65 & 82.8 & 140.6 & 73.6 \\
Oct & 3.95 & 21.06 & 14.96 & 18.01 & 85.8 & 232.0 & 55.1 \\
Nov & 3.14 & 16.31 & 10.52 & 13.41 & 86.4 & 187.9 & 42.1 \\
Dec & 2.89 & 12.23 & 9.55 & 10.9 & 85.5 & 158.2 & 38.7 \\
\hline
\end{tabular}

Table 3. Soil classification based on U.S.D.A soil taxonomy clasifications

\begin{tabular}{lrll}
\hline Physiographic units & Soil series & \multicolumn{2}{c}{ U.S.D.A Soil Taxonomy 2003 } \\
\cline { 2 - 4 } & & \multicolumn{1}{c}{ Family } & \multicolumn{1}{c}{ Subgroup } \\
\hline River alluvial plains & 1 & Fine,mixed,active,thermic & AntheraquicHapludalfs \\
& 2 & Fine,mixed,active,thermic & TypicEndoaqualfs \\
& 3 & Fine,mixed, super active,calcareous, thermic & FluventicEndoaqualfs \\
& 5 & Fine,mixed, super active,calcareous,thermic & TypicEndoaqualfs \\
& 6 & Fine,mixed,active,thermic & FluventicEndoaqualfs \\
River banks & 7 & Fine,mixed, super active,calcareous,thermic & Oxyaquicudiflunents \\
& 8 & Fine,mixed, super active,calcareous,thermic & FluaquenticEpiaquepts \\
Low lands & 9 & Fine,mixed,active,calcareous, thermic & MollicEpiaquepts \\
& 10 & Sandy,mixed, thermic & Oxyaquenticudipsamments \\
Costal lands and & 11 & Fine,mixed, super active,calcareous,thermic & TypicFluvaquents \\
\hline
\end{tabular}

Source: Sys C et al. [1]. 
EC, N, and P) for each land mapping units (LMUs). A total of 11 different LMUs were distinguished and their land use requirements (LURs) were also determined by using expertise's local knowledge, related literature information and available data. The set of LURs are the main defining factors of the LUTs and are used to define physical suitability, increase costs of production or reduce yields of outputs in LMUs. Furthermore, Physiological Crops Requirements (PCRs), based on Sys et al. [4] plant requirements standard database, were collected for each crops.

Land suitability (LS) is a function derived from a set of LURs determined for LUTs and a set of land characteristics (LCs) measured for PCRs, equation 1:

$$
\text { Equation 1: LSI }=\mathrm{f}\{\mathrm{LCs}, \mathrm{PCRs}, \mathrm{CI}\}
$$

The Land Suitability Index (LSI) of the LMUs for each LUT was calculated, using the multiplicative combination of suitability rating index, and LSI values were expressed according to a rating scale of suitability classification for each LUT. At the same time, all LCs were standardized according to the Sys et al. [1] common scale [0...100], where the least beneficial value of LC is 0 and the most suitability is 100. Namely, the restricted nature of each LC is taken into accountby its effect in reducing productivity. Land suitability subclasses ( $w$ for drainage, s for soil, $\mathrm{f}$ for fertility and $\mathrm{c}$ for climatic constrain) reflecting kinds of limitation, or main kinds of improvement measures required, within classes. Based on obtained information about topography (including soil slope), soil (soil depth, soil texture, gypsum and lime content, soil salinity (EC) and alkalinity (ESP) and drainage (water table depth), climate (relative humidity, temperature, rainfall, potential evapo-transpiration and sun radiation during different plant growth stages), properties of soil fertility such as cation exchange capacity (CEC), percentage of basic saturation (PBS), organic carbon (OC\%) and soil acidity $(\mathrm{pH})$ were considered in terms of soil fertility and suitability evaluation methods [20], simple limitation method, and parametric methods (storie methods) were selected.The land suitability classes of 15 common agriculturalcropsharvesting in the paddy fields of Guilan plain under RBCS were determined including rice(Oryzasativa) as the first major crop,andratun(rice)(Oryzasativa), wheat(Triticum aestivum), barley(HordeumVulgare), triticale(Triticale hexaploi), lettuce (Lactuca sativa), carrot(Daucuscarotal.ssp.sativus[Hofdm]), beans(Viciafaba), kidney beans(Phaselus vulgaris), clover (Trifolium alexandrinum), rapeseed (Brassica napus), spinach(Spinaci oleraceae), shallot cress(Lepidum sativum.
L), garlic(Allium sativum) and radish(RhaphanussativusL. var. longipinatus) as the second crops(Table 4).

\section{Results and Discussion}

Suitability is largely a matter of producing crop yield as economic agriculture $[16,17]$. There are two stages offinding a land suited for a specific crop. First, the requirements ofthe crop need to be known, or alternatively which kinds of soil and site attributes adversely influence the crop. The second stage is to identify and delineate land with desirable attributes.

In the present study, specified crops requirements suggested by Sys et al. [16] and Givi [17, 18] were used for rice (as the first crop), ratun(rice as the second crop), wheat, barley, triticale, lettuce, carrot, beans, kidney beans, clover, rape seed, spinach, shallot cress, garlic and radish (the second crops after rice season). Eleven soil series and 24 soil units were derived from the semi-detailed soil study of the central Guilan (Figure 1 and Tables 3 and 4).

Simple Limitation (SL) and parametric methods (Data not shown) were employed in the ultimate evaluation of the quantitative land suitability for different typical land uses. The Results obtained from the physical evaluation showed a close correlation between these two methods. However, due to the interaction of many-sided impacts of the land characteristics, using the SL method will lead to low difference with estimating the obtained land classes compared to the farmers gained (Data not shown). Therefore, in order to fill these gaps, it is strongly recommended that, the land suitability evaluation (especially, the rate of the land and soil properties) should be modified according to the new local studies. Based on the SLM, results (Table 5). There was no optimal climatic condition for irrigated rice production as the first crop and ratun as the second crop in RBCS which makes the region moderately suitable class $\left(\mathrm{S}_{2}\right)$ for rice and marginally suitable class $\left(\mathrm{S}_{3}\right)$ for ratun.

Generally, in the region under consideration, the most important limitation factor for producing rice and ratunin RBCS is climatic characters, especially mean and maximum temperature and $\mathrm{n} / \mathrm{N}$ ratio on growing cycle. The climate character sub-classfor ratun $\left(\mathrm{S}_{3 \mathrm{C}}\right)$ is more serious than rice $\left(\mathrm{S}_{2 \mathrm{C}}\right)$. According to the results, the land units of 1.1 to 8.2 showed the best land suitability for rice (as the first crop). Although in some soil units (9.1 to 11.1), soil and drainage limitation (especially, texture and groundwater table depth) in combination with climatic limitation severely decrease the suitability of study area $\left(\mathrm{S}_{3 \mathrm{cw}}\right.$ or $\left.\mathrm{N}_{1}\right)$. Mahmoud Soltani [7] 
Table 4. Physical and chemical properties of the soil units in the study area

\begin{tabular}{|c|c|c|c|c|c|c|c|c|c|c|c|c|}
\hline $\begin{array}{l}\text { Soil map } \\
\text { unit }\end{array}$ & Horizon & Depth & $\begin{array}{c}\mathrm{pH} \\
\mathrm{H}_{2} \mathrm{O}\end{array}$ & $\left.C^{*}\right)$ & $\mathrm{S}^{*}$ & $\mathrm{Si}^{*}$ & OC & $\mathrm{CaCO}_{3}$ & $\mathrm{ECe}$ & CEC & B.S & Texture \\
\hline & & $\mathrm{cm}$ & & & & $\%$ & & & $\mathrm{dS} / \mathrm{m}$ & $\mathrm{Cmol}+/ \mathrm{kg}$ & $\%$ & \\
\hline \multirow[t]{4}{*}{$1.1^{*}$} & $\mathrm{~A}_{\mathrm{pg}}$ & $0-25$ & 7.27 & 45 & 37 & 18 & 1.87 & 8.5 & 0.47 & 29.12 & 98.2 & $\mathrm{C}$ \\
\hline & $\mathrm{B}_{\mathrm{t}}^{\mathrm{Pg}}$ & $25-70$ & 7.26 & 46.8 & 26.8 & 26.4 & 0.62 & 9 & 0.42 & 26.6 & 99.2 & $\mathrm{C}$ \\
\hline & $\mathrm{B}_{\mathrm{wg} 1}$ & $70-100$ & 7.83 & 42.8 & 21.2 & 36 & 0.08 & 14 & 0.59 & 25 & 99.6 & $\mathrm{C}$ \\
\hline & $\mathrm{B}_{\mathrm{wg} 2}^{\mathrm{wg} 1}$ & $100-150$ & 7.83 & 40.8 & 21.2 & 38 & 0.08 & 20 & 0.59 & 24.9 & 100 & $\mathrm{C}$ \\
\hline \multirow[t]{4}{*}{$2.1^{*}$} & $A_{p}$ & $0-20$ & 7.23 & 38.8 & 30.4 & 30.8 & 1.72 & 19.25 & 0.69 & 26.16 & 100 & CL \\
\hline & $B_{t}^{P}$ & $20-60$ & 7.76 & 44.8 & 13.4 & 41.8 & 0.39 & 21.50 & 0.66 & 26.5 & 100 & $\mathrm{SiC}$ \\
\hline & $\mathrm{B}_{\mathrm{tg}}$ & $60-100$ & 7.67 & 56 & 19.6 & 24.4 & 0.82 & 18.50 & 0.79 & 28.6 & 100 & $\mathrm{C}$ \\
\hline & $B_{w g}^{g}$ & $100-150$ & 7.55 & 45.6 & 12.4 & 40.4 & 0.66 & 15.40 & 0.62 & 24.50 & 100 & $\mathrm{SiC}$ \\
\hline \multirow[t]{5}{*}{$3.1^{*}$} & $A_{p}$ & $0-19$ & 7.55 & 38 & 24.4 & 37.6 & 2.46 & 10 & 1.03 & 28.35 & 99.8 & $\mathrm{CL}$ \\
\hline & $A B$ & $19-40$ & 7.76 & 47.8 & 11 & 41.2 & 1.05 & 13.75 & 0.72 & 24.86 & 100 & $\mathrm{SiC}$ \\
\hline & $\mathrm{B}_{\mathrm{wgl}}$ & $40-64$ & 7.61 & 40.6 & 23.2 & 36.2 & 2.11 & 17.5 & 0.46 & 25.7 & 99.9 & $\mathrm{C}$ \\
\hline & $\mathrm{B}_{\mathrm{wg} 2}$ & $64-90$ & 7.27 & 42.8 & 19.2 & 38 & 3.28 & 19.25 & 0.46 & 27.24 & 99.8 & $\mathrm{C}$ \\
\hline & $\mathrm{BC}_{\mathrm{g}}$ & $90-150$ & 7.15 & 36 & 47 & 17 & 1.95 & 20.5 & 2.15 & 22.1 & 100 & SC \\
\hline \multirow[t]{5}{*}{$4.3^{*}$} & $A_{p}$ & $0-18$ & 6.79 & 35 & 25.8 & 39.2 & 2.57 & - & 0.59 & 22.01 & 100 & CL \\
\hline & $\mathrm{A}_{\mathrm{B}}^{\mathrm{B}}$ & $18-38$ & 7.46 & 49.4 & 18.6 & 32 & 0.62 & - & 0.49 & 23.27 & 100 & $\mathrm{C}$ \\
\hline & $\mathrm{B}_{\mathrm{tg} 1}^{\mathrm{g}}$ & $38-65$ & 7.9 & 51.2 & 20.4 & 28.4 & 0.35 & - & 0.44 & 27.88 & 100 & $\mathrm{C}$ \\
\hline & $\mathrm{B}_{\mathrm{tg} 2}^{\mathrm{g} 1}$ & $65-95$ & 7.78 & 41.8 & 14.2 & 44 & 0.20 & - & 1 & 21.8 & 100 & $\mathrm{SiC}$ \\
\hline & $\mathrm{BC}_{\mathrm{g}}$ & $95-150$ & 8.07 & 37.8 & 49.2 & 13 & 0.20 & - & 0.86 & 19.7 & 100 & SC \\
\hline \multirow[t]{3}{*}{$5.1^{*}$} & $A_{p}$ & $0-20$ & 6.79 & 39.6 & 16.4 & 44 & 5.93 & - & 2.84 & 32.79 & 97.6 & $\mathrm{SiCL}$ \\
\hline & $\mathrm{B}_{\mathrm{wgl}}^{\mathrm{r}}$ & $20-50$ & 7.47 & 45 & 20.6 & 34.4 & 1.99 & - & 1.02 & 24.92 & 100 & $\mathrm{C}$ \\
\hline & $\mathrm{B}_{\mathrm{wg} 2}^{\mathrm{wg} 1}$ & $50-150$ & 7.27 & 46.8 & 21.2 & 32 & 1.72 & - & 0.89 & 16.6 & 100 & $\mathrm{C}$ \\
\hline \multirow[t]{4}{*}{$6.1^{*}$} & $A_{p}$ & $0-35$ & 7.54 & 29.8 & 35.4 & 34.8 & 2.15 & - & 0.58 & 21.3 & 100 & CL \\
\hline & $\mathrm{Bw}_{\mathrm{w}}^{\mathrm{p}}$ & $35-65$ & 7.69 & 30 & 48 & 22 & 0.43 & - & 0.51 & 19.2 & 100 & SCL \\
\hline & $\mathrm{C}$ & $65-90$ & 7.82 & 15.6 & 65.4 & 19 & 0.20 & - & 0.45 & 12 & 100 & SL \\
\hline & $2 \mathrm{~B}$ & $90-150$ & 7.57 & 41.4 & 15.4 & 43.2 & 1.17 & - & 0.45 & 23.14 & 100 & SiL \\
\hline \multirow[t]{6}{*}{$7.2^{*}$} & $A_{p}$ & $0-10$ & 7.37 & 36.8 & 34.4 & 28.8 & 1.05 & 16.2 & 0.73 & 25.34 & 99.7 & CL \\
\hline & $\mathrm{C} 1$ & $10-25$ & 7.55 & 29.8 & 38.2 & 32 & 0.86 & 18.75 & 0.7 & 21.31 & 99.9 & CL \\
\hline & $\mathrm{C} 2$ & $25-50$ & 8 & 10 & 81.2 & 8.8 & 0.04 & 15.75 & 0.37 & 12.35 & 100 & LS \\
\hline & C3 & $50-80$ & 7.76 & 28.8 & 25.6 & 45.6 & 0.62 & 20 & 0.87 & 18.75 & 100 & CL \\
\hline & $2 \mathrm{C}_{\mathrm{g} 1}$ & $80-110$ & 7.54 & 28.2 & 29.8 & 42 & 0.43 & 20 & 1.67 & 16.82 & 100 & CL \\
\hline & $2 C_{g 2}^{g 1}$ & $110-150$ & 7.54 & 9 & 83 & 8 & 0.27 & 16.25 & 2.04 & 13.69 & 100 & LS \\
\hline \multirow[t]{3}{*}{$8.1^{*}$} & $\mathrm{~A}_{\mathrm{pg}}$ & $0-40$ & 7.23 & 39 & 16.8 & 44.2 & 7.96 & - & 1.22 & 32.28 & 100 & $\mathrm{SiCL}$ \\
\hline & $\mathrm{B}_{\mathrm{wg} 1}^{\mathrm{Pb}}$ & $40-80$ & 7.96 & 41.8 & 16.2 & 42 & 0.78 & - & 0.54 & 22.08 & 100 & $\mathrm{SiC}$ \\
\hline & $\mathrm{B}_{\mathrm{wg} 2}$ & $80-120$ & 8.03 & 48.2 & 21.8 & 30 & 0.55 & - & 0.94 & 19.11 & 100 & $\mathrm{C}$ \\
\hline \multirow[t]{5}{*}{$9.1^{*}$} & $A_{p g}$ & $0-10$ & 6.85 & 19 & 23.4 & 57.6 & 11.3 & - & 0.83 & 28.3 & 98.6 & $\mathrm{SiL}$ \\
\hline & $\mathrm{B}_{\mathrm{wgl}}^{\mathrm{Pb}}$ & $10-25$ & 6.61 & 41.8 & 11.8 & 46.4 & 3.12 & - & 0.7 & 30 & 97 & $\mathrm{SiC}$ \\
\hline & $\mathrm{B}_{\mathrm{wg} 2}$ & $25-70$ & 7.53 & 47 & 10.2 & 42.8 & 0.84 & - & 0.38 & 25 & 100 & $\mathrm{SiC}$ \\
\hline & $B_{\text {wg3 }}$ & $70-100$ & 7.53 & 41.6 & 12.8 & 45.6 & 0.82 & - & 0.48 & 24.6 & 100 & $\mathrm{SiC}$ \\
\hline & $\mathrm{C}_{\mathrm{g}}$ & $100-150$ & 7.66 & 47.2 & 12.4 & 40.4 & 0.04 & - & 0.57 & 25 & 100 & $\mathrm{SiC}$ \\
\hline \multirow[t]{3}{*}{$10.1^{*}$} & A & $0-20$ & 5.77 & 9 & 81 & 10 & 0.27 & - & 0.16 & 13.14 & 98.9 & LS \\
\hline & $\mathrm{C}_{1}$ & $20-80$ & 6.89 & 6 & 89.2 & 4.8 & 0.08 & - & 0.17 & 10.89 & 100 & S \\
\hline & $\mathrm{C}_{2}$ & $80-120$ & 8.19 & 6 & 88.4 & 5.6 & nd & - & 0.24 & 9.8 & 100 & S \\
\hline \multirow[t]{6}{*}{$11.1^{*}$} & $A_{p}$ & $0-20$ & 7.52 & 21.2 & 27.4 & 51.4 & 2.15 & 8.25 & 0.88 & 16.1 & 100 & $\mathrm{SiL}$ \\
\hline & $C^{P}$ & $20-40$ & 7.43 & 4 & 88 & 8 & 2.05 & 7.25 & 0.7 & 9.6 & 100 & $S$ \\
\hline & $2 \mathrm{~A}$ & $40-63$ & 7.64 & 26 & 23.2 & 50.8 & 2.11 & 11.5 & 0.77 & 20.1 & 100 & SiL \\
\hline & $2 \mathrm{AB}_{\mathrm{gb}}$ & $63-95$ & 7.67 & 34.4 & 19.8 & 45.8 & 1.83 & 10.25 & 1.2 & 22.2 & 100 & CL \\
\hline & $2 \mathrm{C}_{\mathrm{gb} 1}^{\mathrm{go}}$ & $95-110$ & 7.56 & 45 & 10.2 & 44.8 & 2.11 & 16.75 & 1.19 & 27.1 & 100 & SiL \\
\hline & $2 C_{g b 2}^{g b 1}$ & $110-145$ & 7.6 & 45.4 & 25 & 29.6 & 2.57 & 17.25 & 1.86 & 29.3 & 100 & $\mathrm{C}$ \\
\hline
\end{tabular}




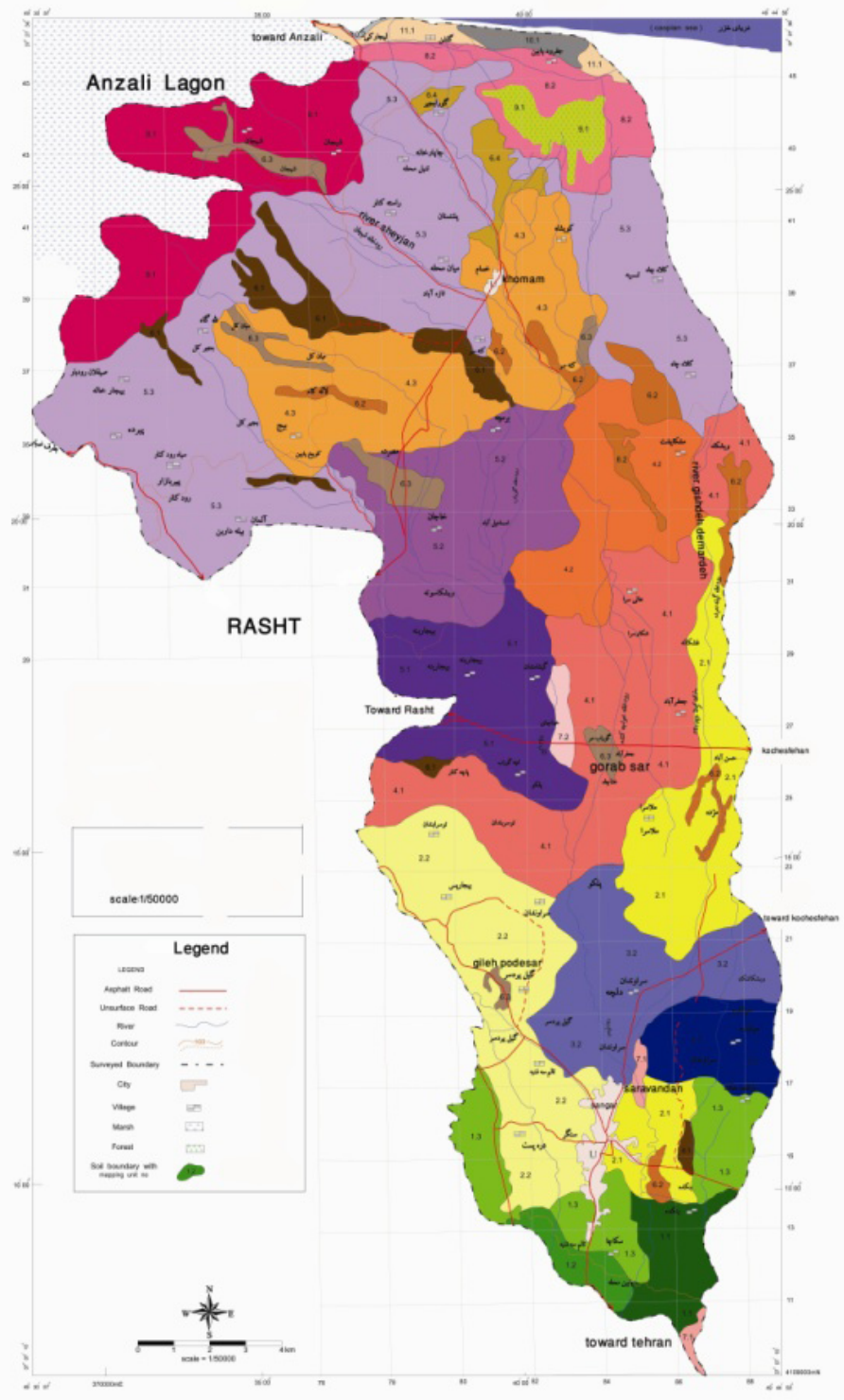

Figure 1. Soil units map of the study area (Central Guilan).

indicated that SLM and PM show similar suitability classes which confirms the previous findings for several crops by other researchers. In many cases, however, the use of SLM revealed to be more realistic in showing the distinguished suitability classes in 4 soil series in the north coastal plain (Amol research station field of rice research institute-Iran). Therefore, based on the obtained results, the cultivation of rice can be classified either as $S_{1}$ with soil, drainage and fertility limitation or as $S_{3 C}$ in combination with climate limitation. Also, Torabi [8] and Akef [10] showed the land suitability class for rice was $S_{2}$ due to soil characters, but $S_{3}$ with combination of climatic requirements.
Irrigated carrot can be evaluated from a marginal suitability subclass $\left(\mathrm{S}_{3 \mathrm{Swc}}\right.$ and $\left.\mathrm{S}_{3 \mathrm{fwc}}\right)$ to unsuitable class $\left(\mathrm{N}_{2}\right)$ because of the mean annual temperature during the growing cycle, low organic carbon content, and low water table depth (high wetness). The soil limitation factor (texture) and drainage limitation (low water table depth) result in moderately suitable $\left(\mathrm{S}_{2}\right)$ to marginally suitable classes for spinach, radish, lettuce, garlic and clover. Ismail et al. [12] stated that land suitability evaluation for rice in $40 \%$ and for vegetables in $47 \%$ of the study area soils are marginally suitable $\left(\mathrm{S}_{3 \mathrm{C}}\right)$ because of climatic (temperature) and soil limitation (texture and structure). 
Table 5. Land suitability classes for representative soil units of the study area for 15 plants

\begin{tabular}{|c|c|c|c|c|c|c|c|c|}
\hline Soil map unit & Rice & Rice & Spinach & Beans & Radish & Triticale & Barley & Garlic \\
\hline 1.1 & $\mathrm{~S}_{2 \mathrm{C}}$ & $\mathrm{S}_{3 \mathrm{C}}$ & $\mathrm{S}_{2 \mathrm{~S}}$ & $\mathrm{~S}_{1}$ & $\mathrm{~S}_{3 \mathrm{~S}}$ & $\mathrm{~S}_{2 \mathrm{~W}}$ & $\mathrm{~S}_{3 \mathrm{C}}$ & $\mathrm{S}_{2 \mathrm{~S}}$ \\
\hline 1.2 & $\mathrm{~S}_{2 \mathrm{C}}$ & $\mathrm{S}_{3 \mathrm{C}}$ & $\mathrm{S}_{2 \mathrm{SW}}$ & $\mathrm{S}_{2 \mathrm{SW}}$ & $\mathrm{S}_{2 \mathrm{SW}}$ & $\mathrm{S}_{2 \mathrm{~W}}$ & $\mathrm{~S}_{3 \mathrm{C}}$ & $\mathrm{S}_{2 \mathrm{SW}}$ \\
\hline 1.3 & $\mathrm{~S}_{2 \mathrm{C}}$ & $\mathrm{S}_{3 \mathrm{C}}$ & $\mathrm{S}_{2 \mathrm{~W}}$ & $S_{2 W}$ & $\mathrm{~S}_{2 \mathrm{~W}}$ & $\mathrm{~S}_{2 \mathrm{~W}}$ & $\mathrm{~S}_{3 \mathrm{C}}$ & $\mathrm{S}_{2 \mathrm{~W}}$ \\
\hline 2.1 & $\mathrm{~S}_{2 \mathrm{C}}$ & $\mathrm{S}_{3 \mathrm{C}}$ & $\mathrm{S}_{2 \mathrm{~W}}$ & $\mathrm{~S}_{2 \mathrm{~W}}$ & $\mathrm{~S}_{2 \mathrm{SW}}$ & $\mathrm{S}_{2 \mathrm{SW}}$ & $\mathrm{S}_{3 \mathrm{C}}$ & $\mathrm{S}_{2 \mathrm{SW}}$ \\
\hline 2.2 & $\mathrm{~S}_{2 \mathrm{C}}$ & $\mathrm{S}_{3 \mathrm{C}}$ & $\mathrm{S}_{2 \mathrm{SW}}$ & $\mathrm{S}_{2 \mathrm{SW}}$ & $\mathrm{S}_{2 \mathrm{SW}}$ & $\mathrm{S}_{3 \mathrm{~W}}$ & $\mathrm{~N}_{1 \mathrm{~W}}$ & $\mathrm{~S}_{2 \mathrm{SW}}$ \\
\hline 3.1 & $\mathrm{~S}_{2 \mathrm{CW}}$ & $\mathrm{S}_{3 \mathrm{C}}$ & $\mathrm{S}_{2 \mathrm{SW}}$ & $\mathrm{S}_{2 \mathrm{~W}}$ & $\mathrm{~S}_{3 \mathrm{~W}}$ & $\mathrm{~S}_{3 \mathrm{~W}}$ & $\mathrm{~S}_{3 \mathrm{WC}}$ & $\mathrm{S}_{2 \mathrm{SW}}$ \\
\hline 3.2 & $\mathrm{~S}_{2 \mathrm{C}}$ & $\mathrm{S}_{3 \mathrm{C}}$ & $\mathrm{S}_{2 \mathrm{SW}}$ & $\mathrm{S}_{2 \mathrm{SW}}$ & $\mathrm{S}_{2 \mathrm{SW}}$ & $\mathrm{S}_{2 \mathrm{SW}}$ & $\mathrm{S}_{3 \mathrm{C}}$ & $\mathrm{S}_{2 \mathrm{SW}}$ \\
\hline 4.1 & $\mathrm{~S}_{2 \mathrm{CW}}$ & $\mathrm{S}_{3 \mathrm{C}}$ & $\mathrm{S}_{2 \mathrm{SW}}$ & $\mathrm{S}_{2 \mathrm{SW}}$ & $\mathrm{S}_{2 \mathrm{SW}}$ & $\mathrm{S}_{3 \mathrm{~W}}$ & $\mathrm{~N}_{1 \mathrm{~W}}$ & $\mathrm{~S}_{2 \mathrm{SW}}$ \\
\hline 4.2 & $\mathrm{~S}_{2 \mathrm{CW}}$ & $\mathrm{S}_{3 \mathrm{C}}$ & $\mathrm{S}_{2 \mathrm{~W}}$ & $\mathrm{~S}_{3 \mathrm{~W}}$ & $\mathrm{~S}_{2 \mathrm{~W}}$ & $\mathrm{~S}_{3 \mathrm{~W}}$ & $\mathrm{~N}_{1 \mathrm{~W}}$ & $\mathrm{~S}_{2 \mathrm{SW}}$ \\
\hline 4.3 & $\mathrm{~S}_{2 \mathrm{CW}}$ & $\mathrm{S}_{3 \mathrm{C}}$ & $\mathrm{S}_{3 \mathrm{~W}}$ & $\mathrm{~S}_{3 \mathrm{~W}}$ & $\mathrm{~S}_{3 \mathrm{~W}}$ & $\mathrm{~S}_{3 \mathrm{~W}}$ & $\mathrm{~N}_{1}$ & $\mathrm{~S}_{2 \mathrm{SW}}$ \\
\hline 5.1 & $\mathrm{~S}_{2 \mathrm{C}}$ & $\mathrm{S}_{3 \mathrm{C}}$ & $\mathrm{S}_{2 \mathrm{SW}}$ & $\mathrm{S}_{3 \mathrm{~W}}$ & $\mathrm{~S}_{2 \mathrm{SW}}$ & $\mathrm{S}_{3 \mathrm{~W}}$ & $\mathrm{~N}_{1}$ & $\mathrm{~S}_{2 \mathrm{SW}}$ \\
\hline 5.2 & $\mathrm{~S}_{2 \mathrm{C}}$ & $\mathrm{S}_{3 \mathrm{C}}$ & $\mathrm{S}_{2 \mathrm{SW}}$ & $\mathrm{S}_{3 \mathrm{~W}}$ & $\mathrm{~S}_{2 \mathrm{SW}}$ & $\mathrm{S}_{3 \mathrm{~W}}$ & $\mathrm{~N}_{1}$ & $\mathrm{~S}_{2 \mathrm{SW}}$ \\
\hline 5.3 & $\mathrm{~S}_{2 \mathrm{CW}}$ & $\mathrm{S}_{3 \mathrm{C}}$ & $\mathrm{S}_{3 \mathrm{~W}}$ & $S_{3 W}$ & $\mathrm{~S}_{3 \mathrm{~W}}$ & $\mathrm{~S}_{3 \mathrm{~W}}$ & $\mathrm{~N}_{1}$ & $\mathrm{~S}_{2 \mathrm{SW}}$ \\
\hline 6.1 & $\mathrm{~S}_{2 \mathrm{C}}$ & $\mathrm{S}_{3 \mathrm{C}}$ & $\mathrm{S}_{25 \mathrm{~W}}$ & $\mathrm{~S}_{3 \mathrm{~W}}$ & $\mathrm{~S}_{2 \mathrm{SW}}$ & $\mathrm{S}_{3 \mathrm{~W}}$ & $\mathrm{~N}_{1}$ & $\mathrm{~S}_{2 \mathrm{SW}}$ \\
\hline 6.2 & $\mathrm{~S}_{2 \mathrm{CS}}$ & $\mathrm{S}_{3 \mathrm{C}}$ & $\mathrm{S}_{2 \mathrm{~W}}$ & $\mathrm{~S}_{2 \mathrm{~W}}$ & $\mathrm{~S}_{2 \mathrm{~W}}$ & $\mathrm{~S}_{2 \mathrm{~W}}$ & $\mathrm{~S}_{3 \mathrm{C}}$ & $\mathrm{S}_{2 \mathrm{~W}}$ \\
\hline 6.3 & $\mathrm{~S}_{2 \mathrm{CW}}$ & $\mathrm{S}_{3 \mathrm{C}}$ & $\mathrm{S}_{3 \mathrm{~W}}$ & $\mathrm{~S}_{3 \mathrm{~W}}$ & $\mathrm{~S}_{3 \mathrm{~W}}$ & $\mathrm{~S}_{3 \mathrm{~W}}$ & $\mathrm{~N}_{1}$ & $\mathrm{~S}_{2 \mathrm{~W}}$ \\
\hline 7.1 & $\mathrm{~S}_{3 \mathrm{~S}}$ & $\mathrm{~S}_{3 \mathrm{C}}$ & $\mathrm{S}_{2 \mathrm{SW}}$ & $\mathrm{S}_{2 \mathrm{SW}}$ & $\mathrm{S}_{2 \mathrm{SW}}$ & $\mathrm{S}_{2 \mathrm{SW}}$ & $\mathrm{S}_{3 \mathrm{C}}$ & $\mathrm{S}_{2 \mathrm{~W}}$ \\
\hline 7.2 & $\mathrm{~S}_{3 \mathrm{~S}}$ & $\mathrm{~S}_{3 \mathrm{C}}$ & $\mathrm{S}_{2 \mathrm{SW}}$ & $\mathrm{S}_{2 \mathrm{SW}}$ & $\mathrm{S}_{2 \mathrm{SW}}$ & $\mathrm{S}_{2 \mathrm{SW}}$ & $\mathrm{S}_{3 \mathrm{C}}$ & $\mathrm{S}_{2 \mathrm{~W}}$ \\
\hline 8.1 & $\mathrm{~S}_{3 \mathrm{~W}}$ & $\mathrm{~S}_{3 \mathrm{WC}}$ & $\mathrm{S}_{3 \mathrm{~W}}$ & $\mathrm{~S}_{3 \mathrm{~W}}$ & $\mathrm{~S}_{3 \mathrm{~W}}$ & $\mathrm{~S}_{3 \mathrm{~W}}$ & $\mathrm{~N}_{1}$ & $\mathrm{~S}_{2 \mathrm{~W}}$ \\
\hline 8.2 & $\mathrm{~S}_{2 \mathrm{CW}}$ & $\mathrm{S}_{3 \mathrm{C}}$ & $\mathrm{S}_{3 \mathrm{~W}}$ & $\mathrm{~S}_{3 \mathrm{~W}}$ & $S_{3 W}$ & $\mathrm{~S}_{3 \mathrm{~W}}$ & $\mathrm{~N}_{1}$ & $\mathrm{~S}_{2 \mathrm{~W}}$ \\
\hline 9.1 & $\mathrm{~N}_{1}$ & $\mathrm{~N}_{1}$ & $\mathrm{~S}_{3 \mathrm{~W}}$ & $\mathrm{~S}_{3 \mathrm{~W}}$ & $\mathrm{~S}_{3 \mathrm{~W}}$ & $\mathrm{~S}_{3 \mathrm{~W}}$ & $\mathrm{~N}_{1}$ & $\mathrm{~S}_{2 \mathrm{~W}}$ \\
\hline 10.1 & $\mathrm{~N}_{1}$ & $\mathrm{~N}_{1}$ & $\mathrm{~S}_{2 \mathrm{SW}}$ & $\mathrm{S}_{3 \mathrm{SW}}$ & $S_{2 W}$ & $\mathrm{~S}_{3 \mathrm{SW}}$ & $\mathrm{N}_{1}$ & $\mathrm{~S}_{2 \mathrm{SW}}$ \\
\hline 10.2 & $\mathrm{~S}_{3 \mathrm{~S}}$ & $\mathrm{~N}$ & $\mathrm{~S}_{3 \mathrm{sW}}$ & $\mathrm{S}_{3 \mathrm{sW}}$ & $\mathrm{S}_{3 \mathrm{SW}}$ & $\mathrm{S}_{3 \mathrm{SW}}$ & $\mathrm{N}_{1}$ & $\mathrm{~S}_{3 \mathrm{~S}}$ \\
\hline 11.1 & $\mathrm{~S}_{3 \mathrm{~S}}$ & $\mathrm{~S}_{3 \mathrm{C}}$ & $\mathrm{S}_{3 \mathrm{~W}}$ & $S_{3 \mathrm{~W}}$ & $S_{3 W}$ & $\mathrm{~S}_{3 \mathrm{~W}}$ & $\mathrm{~N}_{1}$ & $S_{2 W}$ \\
\hline
\end{tabular}

$\mathrm{s}=$ Soil limitation $\mathrm{f}=$ Fertility limitation, $\mathrm{w}=$ Drainage limitation and $\mathrm{c}=$ Climate limitation

Furthermore, the investigations were carried out on irrigated wheat, barley and triticale showed that the climatic limitation such as mean temperature, absolute min temperature during the vegetative stage, leads to reduced land suitability classes more than soil and drainage limitation $\left(\mathrm{S}_{3 \mathrm{wc}}\right.$ to $\mathrm{N}_{1 \mathrm{wc}}$. According to the results, the most important limiting factors for bean and faba bean are the same as other crops, except organic carbon content and $\mathrm{pH}$ thus the land suitability classes of them varied between $\mathrm{S}_{2}$ and $\mathrm{S}_{3}$. Suggested that the wet Caspian lowlands in the two northern provinces of Iran (Gilan and Mazandaran) are the primary growing areas, where heavy rainfall typically facilitates paddy cultivation in these humid, sub-tropical regions (Ayoubi et al. (2002)). In paddy soils of Mazandaran province the most important crops including rice (Oryza sativa), bean (Vicia Faba) and clover (Trifolium repens) defined three crop rotation systems as following: 1) Rice-Follow (R-F) 2) Rice-Clover (R-C) and 3) Rice-Bean (R-B).
Finally, rapeseed shows moderate suitability to unsuitability classes between different limitation rate of soil and drainage. Therefore, major limitation factors for cropping in the central Guilan, depend on the kind of agricultural cropsare climate (low temperature and high rainfall during the vegetative and reproductive growth stages), drainage (shallow water table depth), soil (texture) and fertility (organic carbon content, $\mathrm{pH}$ ) and (Alone or in combination) respectively. These factors adversely influence on the land suitability classes for the first and second crops in RBCS while the intensity of climatic and drainage limitation cause more negative effects than others because of growing seasons (autumn and winter).

\section{Conclusion}

Generally, majority of the area in central Guilan are marginally suitable for rice and the other off-season crops 
Continue of Table 5. Land suitability classes for representative soil units of the study area for 15 plants

\begin{tabular}{|c|c|c|c|c|c|c|c|}
\hline Soil map unit & Shall cress & Clover & Lettuce & Rape seed & Wheat & Bean & Carrot \\
\hline 1.1 & $\mathrm{~S}_{2 \mathrm{~S}}$ & $\mathrm{~S}_{2 \mathrm{~S}}$ & $\mathrm{~S}_{3 \mathrm{~S}}$ & $\mathrm{~S}_{2 \mathrm{~W}}$ & $\mathrm{~S}_{3 \mathrm{C}}$ & $\mathrm{S}_{2 \mathrm{SC}}$ & $\mathrm{S}_{3 \mathrm{SC}}$ \\
\hline 1.2 & $\mathrm{~S}_{2 \mathrm{SW}}$ & $\mathrm{S}_{2 \mathrm{~S}}$ & $\mathrm{~S}_{2 \mathrm{SW}}$ & $\mathrm{S}_{3 \mathrm{~W}}$ & $S_{3 C}$ & $\mathrm{~S}_{2 \mathrm{SWC}}$ & $\mathrm{S}_{3 \mathrm{SWC}}$ \\
\hline 1.3 & $\mathrm{~S}_{2 \mathrm{~W}}$ & $\mathrm{~S}_{2 \mathrm{~W}}$ & $\mathrm{~S}_{2 \mathrm{~W}}$ & $\mathrm{~S}_{2 \mathrm{~W}}$ & $S_{3 C}$ & $\mathrm{~S}_{3 \mathrm{~W}}$ & $\mathrm{~S}_{3 \mathrm{WC}}$ \\
\hline 2.1 & $\mathrm{~S}_{2 \mathrm{~W}}$ & $\mathrm{~S}_{2 \mathrm{~W}}$ & $\mathrm{~S}_{2 \mathrm{~W}}$ & $\mathrm{~S}_{2 \mathrm{~W}}$ & $\mathrm{~S}_{3 \mathrm{C}}$ & $\mathrm{S}_{2 \mathrm{SWC}}$ & $\mathrm{S}_{3 \mathrm{SWC}}$ \\
\hline 2.2 & $S_{2 S W}$ & $S_{2 W}$ & $\mathrm{~S}_{2 \mathrm{SW}}$ & $\mathrm{S}_{3 \mathrm{~W}}$ & $\mathrm{~S}_{3 \mathrm{WC}}$ & $\mathrm{S}_{2 \mathrm{WC}}$ & $\mathrm{S}_{3 \mathrm{WC}}$ \\
\hline 3.1 & $\mathrm{~S}_{2 \mathrm{SW}}$ & $S_{2 W}$ & $\mathrm{~S}_{2 \mathrm{SW}}$ & $S_{2 W}$ & $\mathrm{~S}_{3 \mathrm{WC}}$ & $\mathrm{S}_{3 \mathrm{~W}}$ & $\mathrm{~S}_{3 \mathrm{SWC}}$ \\
\hline 3.2 & $\mathrm{~S}_{2 \mathrm{SW}}$ & $S_{2 W}$ & $\mathrm{~S}_{2 \mathrm{~W}}$ & $S_{2 W}$ & $\mathrm{~S}_{3 \mathrm{C}}$ & $\mathrm{S}_{2 \mathrm{WFC}}$ & $\mathrm{S}_{3 \mathrm{SWC}}$ \\
\hline 4.1 & $\mathrm{~S}_{2 \mathrm{SW}}$ & $\mathrm{S}_{2 \mathrm{~W}}$ & $\mathrm{~S}_{2 \mathrm{~W}}$ & $\mathrm{~S}_{3 \mathrm{~W}}$ & $\mathrm{~S}_{3 \mathrm{WC}}$ & $\mathrm{S}_{3 \mathrm{~W}}$ & $\mathrm{~S}_{3 \mathrm{SWC}}$ \\
\hline 4.2 & $S_{2 W}$ & $S_{2 \mathrm{~W}}$ & $S_{2 W}$ & $S_{3 W}^{5 W}$ & $\mathrm{~S}_{3 \mathrm{WC}}$ & $S_{3 \mathrm{~W}}$ & $S_{3 S W C}$ \\
\hline 4.3 & $\mathrm{~S}_{3 \mathrm{~W}}$ & $\mathrm{~S}_{3 \mathrm{~W}}$ & $\mathrm{~S}_{3 \mathrm{~W}}$ & $\mathrm{~N}_{1}$ & $\mathrm{~S}_{3 \mathrm{WC}}$ & $\mathrm{S}_{3 \mathrm{~W}}$ & $\mathrm{~S}_{3 \mathrm{SWC}}$ \\
\hline 5.1 & $\mathrm{~S}_{2 \mathrm{SW}}$ & $\mathrm{S}_{2 \mathrm{~W}}$ & $\mathrm{~S}_{2 \mathrm{~W}}$ & $\mathrm{~S}_{3 \mathrm{~W}}$ & $\mathrm{~S}_{3 \mathrm{WC}}$ & $\mathrm{S}_{3 \mathrm{~W}}$ & $\mathrm{~S}_{3 \mathrm{SWC}}$ \\
\hline 5.2 & $\mathrm{~S}_{2 \mathrm{SW}}$ & $S_{3 W}$ & $\mathrm{~S}_{2 \mathrm{~W}}$ & $\mathrm{~S}_{3 \mathrm{~W}}$ & $\mathrm{~S}_{3 \mathrm{WC}}$ & $\mathrm{S}_{3 \mathrm{~W}}$ & $\mathrm{~N}_{1}$ \\
\hline 5.3 & $\mathrm{~S}_{3 \mathrm{~W}}$ & $\mathrm{~S}_{3 \mathrm{~W}}$ & $\mathrm{~S}_{3 \mathrm{~W}}$ & $\mathrm{~N}_{1}$ & $\mathrm{~S}_{3 \mathrm{WC}}$ & $\mathrm{S}_{3 \mathrm{~W}}$ & $\mathrm{~N}_{1 \mathrm{~W}}$ \\
\hline 6.1 & $\mathrm{~S}_{2 \mathrm{SW}}$ & $\mathrm{S}_{2 \mathrm{~W}}$ & $\mathrm{~S}_{2 \mathrm{SW}}$ & $\mathrm{S}_{3 \mathrm{~W}}$ & $\mathrm{~S}_{3 \mathrm{WC}}$ & $\mathrm{S}_{3 \mathrm{~W}}$ & $\mathrm{~S}_{3 \mathrm{WC}}$ \\
\hline 6.2 & $\mathrm{~S}_{2 \mathrm{~W}}$ & $\mathrm{~S}_{2 \mathrm{~W}}$ & $\mathrm{~S}_{2 \mathrm{~W}}$ & $S_{2 W}$ & $\mathrm{~S}_{3 \mathrm{C}}$ & $\mathrm{S}_{3 \mathrm{~W}}$ & $\mathrm{~S}_{3 \mathrm{WC}}$ \\
\hline 6.3 & $\mathrm{~S}_{3 \mathrm{~W}}$ & $\mathrm{~S}_{3 \mathrm{~W}}$ & $\mathrm{~S}_{3 \mathrm{~W}}$ & $\mathrm{~N}_{1}$ & $\mathrm{~S}_{3 \mathrm{WC}}$ & $\mathrm{S}_{3 \mathrm{~W}}$ & $\mathrm{~S}_{3 \mathrm{WC}}$ \\
\hline 7.1 & $\mathrm{~S}_{2 \mathrm{SW}}$ & $\mathrm{S}_{2 \mathrm{~S}}$ & $\mathrm{~S}_{2 \mathrm{~W}}$ & $\mathrm{~S}_{2 \mathrm{~W}}$ & $\mathrm{~S}_{3 \mathrm{C}}$ & $\mathrm{S}_{3 \mathrm{~W}}$ & $\mathrm{~S}_{3 \mathrm{WC}}$ \\
\hline 7.2 & $\mathrm{~S}_{2 S \mathrm{~W}}$ & $\mathrm{~S}_{2 \mathrm{~S}}$ & $\mathrm{~S}_{2 \mathrm{~W}}$ & $S_{2 W}$ & $\mathrm{~S}_{3 \mathrm{C}}$ & $\mathrm{S}_{3 \mathrm{~W}}$ & $\mathrm{~S}_{3 \mathrm{WC}}$ \\
\hline 8.1 & $\mathrm{~S}_{3 \mathrm{~W}}$ & $\mathrm{~S}_{3 \mathrm{~W}}$ & $\mathrm{~S}_{3 \mathrm{~W}}$ & $\mathrm{~N}_{1}$ & $\mathrm{~S}_{3 \mathrm{WC}}$ & $\mathrm{S}_{3 \mathrm{~W}}$ & $\mathrm{~N}_{1}$ \\
\hline 8.2 & $S_{3 W}$ & $S_{3 W}$ & $S_{3 W}$ & $\mathrm{~N}_{1}$ & $\mathrm{~S}_{3 \mathrm{WC}}$ & $S_{3 W}$ & $\mathrm{~N}_{1}$ \\
\hline 9.1 & $\mathrm{~S}_{3 \mathrm{~W}}$ & $\mathrm{~S}_{3 \mathrm{~W}}$ & $\mathrm{~S}_{3 \mathrm{~W}}$ & $\mathrm{~N}_{1}$ & $\mathrm{~S}_{3 \mathrm{WC}}$ & $\mathrm{S}_{3 \mathrm{~W}}$ & $\mathrm{~N}_{1}$ \\
\hline 10.1 & $\mathrm{~S}_{2 \mathrm{SW}}$ & $\mathrm{S}_{3 \mathrm{~S}}$ & $S_{2 W}$ & $\mathrm{~S}_{3 \mathrm{SW}}$ & $\mathrm{S}_{3 \mathrm{C}}$ & $\mathrm{S}_{3 \mathrm{SW}}$ & $\mathrm{S}_{3 \mathrm{FWC}}$ \\
\hline 10.2 & $\mathrm{~S}_{3 \mathrm{SW}}$ & $\mathrm{S}_{2 \mathrm{SW}}$ & $\mathrm{S}_{3 \mathrm{SW}}$ & $\mathrm{S}_{3 \mathrm{SW}}$ & $\mathrm{S}_{3 \mathrm{SWC}}$ & $\mathrm{S}_{3 \mathrm{SWF}}$ & $\mathrm{S}_{3 \mathrm{FWC}}$ \\
\hline 11.1 & $\mathrm{~S}_{3 \mathrm{~W}}$ & $\mathrm{~S}_{3 \mathrm{~W}}$ & $\mathrm{~S}_{3 \mathrm{~W}}$ & $\mathrm{~N}_{1}$ & $\mathrm{~S}_{3 \mathrm{WC}}$ & $\mathrm{S}_{3 \mathrm{~W}}$ & $\mathrm{~N}_{1}$ \\
\hline
\end{tabular}

Subclass symbolss $=$ Soil limitation, $\mathrm{f}=$ Fertility limitation, $\mathrm{w}=$ Drainage limitation and $\mathrm{c}=$ Climate limitation

growing.Therefore, the development efforts (second crops income) should be directed towards realizing this potential.

In general the most limiting factors in economic production of all crops in the study area were composed of soil and drainage, and fertility constrains especially $\mathrm{pH}$ and partly drainage except rice and ratun. Although climatic factors such as high rain fall and temperature caused a marginaly suitable class for the formers crops in comparison by soil and drainage limiting factores suitable class of them $\left(\mathrm{S}_{1}\right)$.

However, this research highlighted the suitability of the area for crops growing, it is important to have verification of the factors considered and their relative significance in informing the decision on suitability. Therefore, to achieve an optimal suitability determination for rice growing under different climatic conditions in Iran, an optimization of the values should be adopted.

\section{References}

1. Sys C, Vanranst E et al. (1991b). Land evaluation part 11: methods in land evaluation, General administration for development cooperation, Agricultural Publications No. 7, Brussels, Belgium.
2. Jafarzadeh A A, Abbasi G (2006). Qualitative land suitability evaluation for the growth of onion, potato, maize, and alfalfa on soils of the Khalat pushan research station, Biologia, vol 61(19), 349-352.

3. F. A. O (1976). A frame work for land evaluation, Soil Bulletin, vol 32, FAO, Rome.

4. Sys C, Vanranst E et al. (1991a). Land evaluation part 1: principles in land evaluation and crop production calculation, General Administration for development cooperation, Agriculture publication No. 7. Brussels, Belgium, 174 pp.

5. Rossiter D G (1994). Lecture notes: Land evaluation, Cornell University, College of Agriculture and Life Sciences, Department of Soil, Crop and Atmospheric Sciences.

6. SeyedJalali S A (2001). Comparison of land suitability classification methods for irrigated winter wheat, Iranian Journal of Soil and Water, Special issue on soil survey and land evaluation, 56-65.

7. Soltani Sh M (2009). Genesis, classification, quantitative and land suitability of Amol research wetland field for rice and second crops, Rice Research Institute of Iran, Rasht, Iran.

8. Torabi H (2001). Genesis, classification, quantitative and qualitative land suitability of eastern of Guilan wetland for rice, $\mathrm{PhD}$ thesis on soil science, Esfehan University.

9. Khaksarian F (2003). Land suitability evaluation of rice at Sabaab plain in Ilam provience, $8 \mathrm{Th}$. Soil congress of Iran, Rasht, Iran. 
10. Akef M (2005). Semi-detailed soil classification of G4 unit of Sepidrood irrigation and drainage network, Local water company of Guilan provience, Guian, Iran.

11. Kuria D, Ngari D et al. (2008). A physical evaluation of land suitability for rice crop using geographical information system: a case study of the tana delta, $3^{\text {rd }}$ ESRI Eastern Africa User Conference (EAUC), Nairobi, Kenya.

12. Ismail H H, Surati J et al. (2002). Evaluation of land suitability for selected land utilization types using geographic information system technology (Case study in Ban gung basin west Java), J.Mana.Hut.Trop, vol 8(2), 11-26.

13. U.S.D.A. (2003a). Soil survey manual, University press of pacific, Honolulu, Hawaii.

14. Klute A (1980). Method of soil analysis. Part I. Physical and chemical and mineralogical methods. American Society Agronomy, U. S. A.

15. U.S.D.A. (2003b). Keys to soil taxonomy, Soil Survey Staff, USDA.

16. Sys C, Vanranst E et al. (1993). Land evaluation part III: Crop Requirements. International Training Center for Post Graduate Soil Scientists, Ghent University, Ghent, Belgium.
17. Givi J (1997). Qualitative evaluation of land suitability for field and fruit crops, $1^{\text {st }}$ Edn., Soil Bulletin No. 1015, Iranian Soil and Water Institute, Tehran, Iran.

18. Givi J (1996). Agricultural growth requirements (Climatic and land properties), Iranian Soil and Water Institute, Tehran, Iran.

19. Jafarzadeh A A, and Atabakazar M R (2004). Land suitability evaluation of Shakaryazi region for wheat, barley, alfalfa, sugar beet, potato and sunflower, Int. Conference of CIGR, Collection of extended abstracts, Session IV, part 5, No. 7, 1-5.

20. Sys C, Vanranst E et al. (1991b). Land evaluation part 11: methods in land evaluation, General Administration for development cooperation, Agricultural Publications No. 7. Brussels, Belgium.

21. Liu W, Qin Y et al. (2006). Land Evaluation in Danling county, Sichuan province, China, 26th Course Professional Master, Geometric and Natural Resources Evaluation, IAO, Florence, Italy, Available from: http://www.iao.florence.it/ training/geomatics/Danling/China_26.pdf

22. Aliasgharzad N, Neyshabouri M R et al. (2006). Effects of arbuscular mycorrhizal fungi and Bradyrhizobium japonicum on drought stress of soybean, Biologia, vol 61(Supl. 19), S324-S328. 